\title{
Autoregulated Heavy Slow Resistance Training Combined with Radial Shockwave Therapy for Plantar Heel Pain: Protocol for a Mixed Methods Pilot Randomised Controlled Trial
}

Ian Burton MSc, CSCS

Physiotherapist, Angus HSCP, NHS Tayside, Dundee, United Kingdom

Please cite as: Burton, I. (2020). Autoregulated Heavy Slow Resistance Training Combined with Radial Shockwave Therapy for Plantar Heel Pain: Protocol for a Mixed Methods Pilot Randomised Controlled Trial. SportRxiv doi: $10.31236 /$ osf.io/ph8bc

\section{ABSTRACT}

Background: Plantar heel pain (PHP) is considered a tendinopathy and it affects up to $10 \%$ of the population. Both heavy slow resistance training (HSRT) and extracorporeal shockwave therapy (ESWT) have shown effectiveness for treating PHP in isolation. However more comprehensive exercise protocols and progression methods are needed due to poor long-term outcomes, and better standardization of ESWT protocols are required. Autoregulation of resistance training involves selfselecting exercise dosage based on individual factors. Although autoregulation has 
proven effective for strength gains in athletes, it has not been investigated in tendinopathy. It is recommended that PHP not be treated by one treatment in isolation, with there being a need to investigate feasibility and effectiveness of combined treatments in PHP. Currently, no studies have investigated autoregulated HSRT combined with ESWT, despite their individual efficacy. The optimal treatment protocol for PHP is unknown, and there is a need to ascertain whether the addition of ESWT to autoregulated HSRT leads to better outcomes compared to either alone. Methods: A three-arm randomised controlled trial (RCT) comparing these groups would be the ideal way to investigate this question, with a pilot RCT testing trial procedures and process evaluation required prior to a definitive RCT. Patients expectations, feasibility and acceptability of combined ESWT and exercise for PHP also remain unknown. Therefore, the addition of qualitative interviews in a mixed methods pilot RCT would help ascertain acceptability and help explain the intervention outcomes.

Keywords: Extracorporeal Shockwave Therapy: Resistance Training; Tendinopathy; Fasciitis, Plantar.

\section{Background}

Musculoskeletal disorders have recently been identified as a leading global cause of disability and pain, comprising $6.7 \%$ of the total global disability-adjusted life years and the second highest (21.3\%) global volume of years lived with disability [1]. Musculoskeletal disorders can have a high morbidity for individuals and represent a vast burden to society and healthcare systems, with effective clinical treatments and recommendations lacking [2]. In the United Kingdom, musculoskeletal disorders are responsible for $30 \%$ of all years lived with disability, and account for $25 \%$ of all GP consultations [3]. Plantar heel pain (PHP) is one of the most common musculoskeletal disorders, which is responsible for $15 \%$ of all clinical foot symptoms, with an individual lifetime prevalence of $10 \%$ in the general population [4]. Although inflammation of the plantar fascia may be present, repetitive microtrauma of the plantar fascia connective tissue leading to chronic degeneration is the likely cause [5]. Therefore, the terms PHP or 'plantar fasciopathy' are now preferred to 'plantar fasciitis' as the disorder is not caused 
by acute inflammation, but is degenerative in nature, presenting like tendinopathy [6]. Symptoms include heel pain in the morning or after activity and functional limitations such as impaired gait leading to reduced physical activity levels [7]. Despite inconclusive evidence on effectiveness, common treatment approaches include taping, manual therapy, ultrasound, low-level laser therapy, orthotics, nonsteroidal anti-inflammatory drugs, corticosteroid injections, and autologous blood-derived injections such as platelet-rich plasma [8]. However, recent systematic reviews suggest that exercise-based approaches and extracorporeal shockwave therapy (ESWT) have the best evidence of effectiveness for treating PHP [9].

Plantar-fascia specific stretching and heavy slow resistance training (HSRT) interventions have been found to be the most effective exercise-based treatments for improving pain and function in PHP, with HSRT being most superior $[10,11]$. Recently, it was found that a patient autoregulated or 'self-dosed' HSRT intervention had similar outcomes to the original standardised protocol, suggesting autoregulated methods may be preferable and more convenient for patients [6]. Autoregulation refers to individuals self-selecting the exercise dosage based on their individual circumstances, exercise response and readiness to train, rather than following a fixed protocol [59]. Although autoregulation has been found more effective than fixed training protocols for increasing strength and performance in athletes, there has been a dearth of research on autoregulation in musculoskeletal physiotherapy, until recently $[6,59]$.

After first being introduced in urology to disintegrate kidney stones, ESWT is now therapeutically applied to a broad range of medical disorders, with clinical effectiveness demonstrated for musculoskeletal conditions such as tendinopathies [12]. Recalcitrant PHP, which is unresponsive to conservative treatment such as exercise, can be treated effectively and safely with ESWT [13]. A large evidencebase now exists demonstrating the effectiveness of radial ESWT (R-ESWT) for PHP, with the body of evidence larger than for any other musculoskeletal disorder [14]. Stretching combined with radial ESWT has also been found more effective than ESWT alone in treating PHP [15]. Recently, rehabilitation consisting of HSRT 
combined with corticosteroid injection was found more effective than either alone for PHP, providing further evidence for combined treatments [16]. Recent research recommends that effective treatments not be delivered in isolation but are combined when treating PHP, as although short-term outcomes are positive, longterm outcomes are often unsatisfactory [6].

Currently, no studies have investigated autoregulated HSRT and ESWT as a combined intervention, despite their individual efficacy. Despite the increasing evidence for ESWT and specific exercises for PHP, the optimal treatment protocol is unknown [17]. In clinical practice, exercise is the most common treatment for PHP and when ESWT is used, it is often combined with exercise. Subsequently, there is a need to ascertain whether the addition of ESWT to HSRT leads to better outcomes compared to either alone. A three-arm randomised controlled trial (RCT) comparing these groups would be the ideal way to investigate this question, with a pilot RCT testing trial procedures and process evaluation required for a definitive RCT. Patients expectations, feasibility and acceptability of ESWT and exercise for PHP also remain unknown. Therefore, the addition of qualitative interviews in a mixed methods pilot RCT would help ascertain acceptability and help explain the intervention outcomes.

\section{Methods}

\section{Study design}

This study (figure 1) will be an explanatory sequential mixed methods pilot RCT, testing recruitment, retention, trial procedures and process evaluation of a largerscale definitive RCT. The quantitative arm is a single centre pilot RCT with three intervention groups (1. ESWT and exercise combined, 2. Exercise alone, 3. ESWT alone), with baseline and two periods of post-intervention assessment; 1-month and 6-months follow-up. The pilot RCT will be followed by semi-structured interviews immediately after intervention completion. The 12-week intervention period has been chosen as it is the same timeframe used in the previous exercise 
intervention RCTs for PHP, allowing enough time for physiological adaptations to occur in response to resistance training $[10,11]$.

\section{Aims}

The primary aim of this study is to pilot the proposed methods, testing recruitment, retention, trial procedures and process evaluation of a larger-scale RCT. This will allow understanding of what worked and why in the intervention implementation and contribute to interpretation of preliminary effectiveness. This method of pilot testing and process evaluation prior to a larger-scale RCT follows the Medical Research Council's (MRC) recommendations for developing and evaluating complex interventions and should provide insights to aid implementation [18, 19]. A secondary aim is to undertake a preliminary comparison of outcome measures and to estimate preliminary effectiveness and the number of participants required to adequately power a full-scale RCT.

\section{Objectives}

Based on the aims of the research, the study objectives are to:

1. Evaluate recruitment and retention rates

2. Evaluate acceptability and feasibility of recruitment methods

3. Evaluate trial procedures

4. Assess feasibility of intervention protocols

5. Evaluate acceptability of interventions to participants

6. Obtain an estimate of the outcome variance to inform sample size calculations for a definitive RCT.

7. Assess secondary outcome measure burden, completion and return rates to confirm whether measures will be completed, and data can be collected to inform acceptability and selection of measures for a definitive RCT.

8. Assess process evaluation methods including intervention fidelity methods to monitor participant and physiotherapist adherence. 
9. Evaluate the use of resources and costs associated with intervention delivery to provide an economic evaluation framework for a definitive RCT.

10. Based on outcomes, design a larger-scale RCT which would be adequately powered to evaluate the clinical and cost-effectiveness of ESWT, autoregulated HSRT and both combined for PHP.

\section{Study setting and Recruitment}

The clinical site will be a private physiotherapy outpatient clinic based in the United Kingdom. Participant recruitment will initially be from the general population via the private physiotherapy clinic. In the event of inadequate recruitment, National Health Service (NHS) services will be considered as an alternative recruitment method. Interested participants will be recruited through convenience sampling via telephone assessment based on inclusion and exclusion criteria. After gaining consent from the assessing clinician, eligible participants meeting inclusion criteria will be posted study information materials. Consenting participants will be invited to the private clinic to undergo clinical assessment by the research physiotherapist to ensure diagnosis of PHP and study consent. Participants will also complete outcome measures to establish baseline pain, function and quality of life (QoL). Demographic and personal information will also be collected at this time. After baseline measures are completed patients will be randomly allocated to one of the three intervention groups by an independent assessor. Selected outcome measures will be repeated at 1 -month and 6-months follow-up via postal delivery. Electronic and postal reminders will be sent to participants to help ensure outcome measure completion. Clinical research must be of benefit participants, such as receiving treatment in experimental RCTs [20]. To achieve this, the proposed study has no groups receiving sham treatment or placebo. Each group is receiving a treatment that has been shown to be effective for PHP. Recruited participants will be informed of the study purpose, potential risks, and provided written informed consent. With ESWT, there is a small risk of temporarily increased pain, which patients' will be informed about [13]. Confidentiality will be maintained by ensuring participants and treatment groups are prepared and concealed in envelopes, which are securely stored [20]. 
Figure 1: Study flow-chart

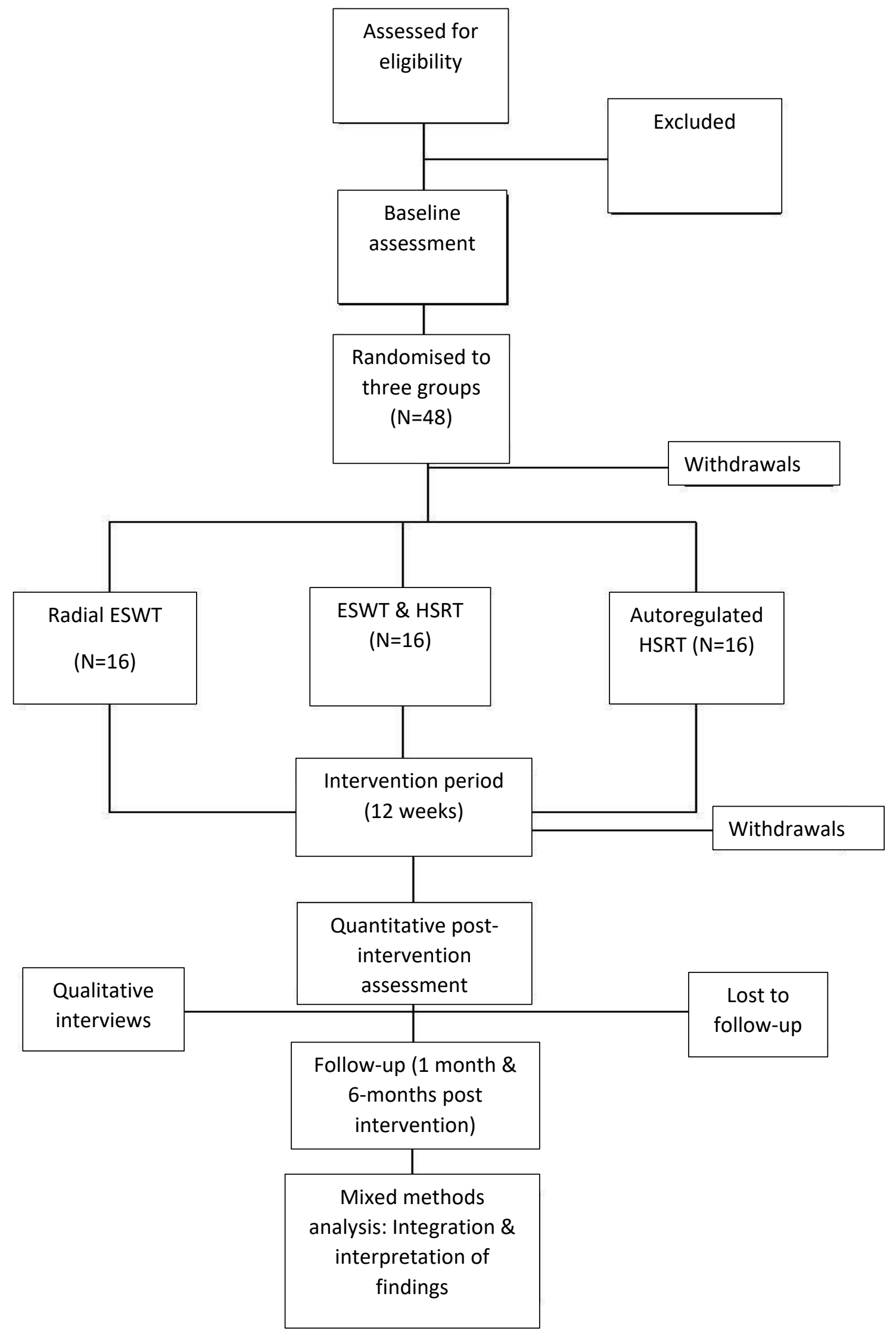


Table 1: Inclusion and exclusion criteria

Inclusion criteria

1. Adults over the age of 18

2. Diagnosis of plantar heel pain by referring clinician to include:

- Heel Pain in the morning or after sitting for extended periods

- Localised pain on palpation where the plantar fascia attaches to the heel - medial calcaneal tuberosity

- Increased pain with walking or standing for extended periods

3. History of at least 3 months of heel pain prior to beginning trial

4. Willingness to abstain from any other treatments during the trial

5. Signed informed consent form

Exclusion criteria

1. Neurological abnormalities, peripheral neuropathy or nerve entrapment syndromes (e.g., tarsal tunnel syndrome)

2. Infections or tumours of the lower extremity, active malignancy

3. Vascular abnormality (e.g., severe varicosities, ischaemia)

4. Arthrosis or arthritis of the foot, rheumatoid arthritis

5. Systemic inflammatory disease, osteomyelitis

6. Dysfunction of the foot or ankle (e.g. chronic instability)

7. Corticosteroid injection or shockwave therapy within 6 weeks of beginning trial interventions

8. Calcaneal stress fracture, plantar fascia rupture or previous surgery for heel spur or plantar fascia

9. Bleeding disorder or haemophilia or active coagulation therapy

10. Cardiac pacemaker

11. Pregnancy 


\section{Participants}

The inclusion and exclusion criteria (Table 1) have been developed based on guidelines and current best practice in the literature from recent RCTs investigating the effects of ESWT and HSRT for PHP [15].

\section{Sample size}

As this is a pilot study primarily testing trial procedures, a formal sample size calculation is not required [21]. The minimum target sample size per intervention group is 12 participants for the pilot RCT, based on recommendations for pilot studies [22]. To help ensure this target is met and allow for potential participant attrition rates, the study will aim to recruit 30\% above this target, which is 16 participants per group [23]. This should give robust and useful information to inform a sample size calculation for a future trial [21].

\section{Blinding and Randomisation}

Given the nature of the three treatment interventions it is not possible to blind participants and physiotherapists to group allocation [24]. However, the research physiotherapist will be blinded to ESWT group allocation by restricting access to allocation records and asking participants not to reveal their group allocation to the assessor [25]. An independent physiotherapist will instruct participants on the autoregulated resistance training protocol and its progression methods and conduct the ESWT interventions following a standardized protocol. An independent assessor will inform participants of group allocation after conducting randomisation using a web-based randomisation service with random variable block-size, with secure password and protected login [26]. The same independent assessor will assess data from outcome measures. 


\section{Interventions}

The R-ESWT machine used in this study will be an Electro Medical Systems (EMSNyon, Switzerland) Swiss DolorClast, with treatment administered by an independent clinic physiotherapist. An independent physiotherapist will also be conducting the HSRT exercise interventions. The independent physiotherapist will interact with study participants strictly in a standardized way irrespective of treatment allocation, preventing any behaviour that could have indicated to the researcher whether participants received R-ESWT treatment. In recent years, RESWT has become increasingly common, leading to the development of widely accepted R-ESWT treatment protocols [15]. Before the intervention, the point of maximum tenderness will be clinically located by the treating physiotherapist, and the handpiece coupled to the identified area by using specific ultrasound coupling gel (EMS Electro Medical Systems). The intervention will be performed in the clinic by the nonblinded physiotherapist, locating the tip of the applicator to the most tender point at the medial calcaneal tubercle, controlling proper placement by patient-controlled feedback, and adjusted during treatment if necessary. With use of the principle of clinical focusing, the area of maximal tenderness will be treated in a circumferential pattern, starting at the point of maximum pain level [15].

Participants in ESWT groups (1 \& 3) will receive three treatments, at weekly intervals as recommended. At each session, 2000 impulses will be applied with an air pressure of 4 bar (equal to a positive energy flux density of $0.16 \mathrm{~mJ} / \mathrm{mm}^{2}$ ). The total positive energy flux density per treatment will be $320 \mathrm{~mJ} / \mathrm{mm}^{2}$, with a treatment frequency of 8 impulses per second [14]. Based on these recommended parameters, each R-ESWT treatment time should last just over four minutes. No anaesthesia will be used as this has been shown to diminish effectiveness of RESWT for PHP [27]. Details of the content of each treatment session and of any adverse effects will be reported on standardized forms [15]. The exercise protocol (Table 2) on autoregulated HSRT will be performed similar to that recommended in the previous RCT [6]. The independent physiotherapist will first instruct and then evaluate each participant in groups 1 and 2 to ensure they are performing the exercises correctly at the first session (week 1 ). Exercise groups (1 and 2) will 
be re-evaluated at week two and three by the physiotherapist to ensure exercise technique and correct progression of HSRT exercise. To reduce confounding variables, all three groups will receive three clinic-based sessions at weekly intervals for three weeks with the same physiotherapist.

Autoregulated HSRT will consist of unilateral heel raises with a towel inserted under the toes to further activate the windlass mechanism [11]. Participants will be instructed to do the exercise on a stairway, step or similar location. The towel is individualized, ensuring participants have their toes maximally dorsal flexed at the top of the heel rise. Participants are instructed to perform the exercise every second day for 12 weeks. Every heel rise consists of a 3-second concentric phase and a 3-second eccentric phase with a 2-second isometric phase at the top. The HSRT will be progressed individually throughout the trial starting with an 8repetition maximum (RM) for five sets. This is defined as the maximal amount of weight that the patient can lift 8 times through the full range of motion while maintaining proper form [6]. If participants cannot perform the required number of repetitions, they are instructed to start the exercises using both legs until they are strong enough to perform unilateral heel raises. They are instructed to keep adding books to the backpack as they became stronger and to record in their diary the amount used, and number completed. In the case of bilateral pain, they are instructed to perform the HSRT with both limbs [11].

Recently, a resistance training specific Rating of Perceived Exertion (RPE) scale has been demonstrated to be effective for measuring effort during a training session and subsequently adjusting load [60]. The scale measures Repetitions in Reserve (RIR), where the individual estimates the amount of additional repetitions remaining at a given load. Although this resistance training specific scale has been validated in weightlifters, its application in rehabilitation has not been evaluated [61]. Nevertheless, this training specific RIR-based RPE scale is a potential method to autoregulate training load in patients prescribed resistance training, such as HSRT programs in tendinopathy [62]. The specific RIR based RPE autoregulation model has been found to lead to greater strength improvements and an overall higher training intensity compared to pre-determined programs in athletes [63- 
64]. The specific RIR-based RPE method of autoregulation could be a potential strategy to ensure that an adequate and optimal amount of load is achieved and progressed for each individual patient in PHP rehabilitation, which is currently not being achieved [6]. The RIR scale will be used in this study as an autoregulation method to self-progress the HSRT and guide loading parameters, with progression and loading criteria detailed in table 2 below.

Table 2: Load adjustment during autoregulated heel-raise resistance training based on rating of percieved exertion and corresponding repeitions in reserve vales.

\begin{tabular}{|c|c|c|c|}
\hline \multicolumn{4}{|c|}{$\begin{array}{l}\text { Warm-up if required } \\
\text { Set } 1 \text { : Based on load lifted during last set of last session }\end{array}$} \\
\hline RPE & RIR & Description of perceived effort & Load adjustment \\
\hline 10 & 0 & $\begin{array}{l}\text { Maximum effort, cannot increase load or } \\
\text { repetitions }\end{array}$ & $-5 \mathrm{~kg}$ \\
\hline 9.5 & 0.5 & $\begin{array}{l}\text { Cannot complete more repetitions but } \\
\text { could increase load }\end{array}$ & $-2.5 \mathrm{~kg}$ \\
\hline 9 & 1 & 1 repetition remaining & Maintain load \\
\hline 8.5 & 1.5 & $1-2$ repetitions remaining & Maintain load \\
\hline 8 & 2 & 2 repetitions remaining & Maintain load \\
\hline 7.5 & 2.5 & 2-3 repetitions remaining & $+2.5 \mathrm{~kg}$ \\
\hline 7 & 3 & 3 repetitions remaining & $+2.5 \mathrm{~kg}$ \\
\hline $5-6$ & $4-6$ & 4-6 repetitions remaining & $+5 \mathrm{~kg}$ \\
\hline $3-4$ & $6+$ & Light effort & $+7.5 \mathrm{~kg}$ \\
\hline $1-2$ & $10+$ & Little to no effort & $+10 \mathrm{~kg}$ \\
\hline
\end{tabular}

Abbreviations: RPE: rating of perceived exertion; RIR: repetitions in reserve; $\mathrm{Kg}$ : Kilograms. 
Low exercise adherence is a common physiotherapy barrier and using a small number of exercises has been shown to result in improved; monitoring, timesaving, self-efficacy, self-management and adherence [28]. The exercise interventions will be delivered by the independent physiotherapist at the clinical site on three occasions over three weeks, with participants continuing them at home over 12 weeks. Written exercise instruction will be given to patients in both exercise groups, including pictures and video links of the exercises together with a description of progression. Patients will also be asked to keep an electronic or written exercise diary to monitor adherence. Participants will also be asked to record in the diary the intake of any pain medication during the study, the number of tablets taken, and the day on which the tablets were taken, as well as any other kind of medication taken during the study [15]. Participants will be asked to use paracetamol within recommended guidelines if pain levels are unacceptable and will be discouraged from using NSAIDs such as ibuprofen and diclofenac as these could diminish treatment effectiveness [29]. All three groups will receive an information sheet on; PHP pathology, pain management, medication, activity modification, and how and when to return slowly to activity.

Before the start of the trial, the research physiotherapist will meet with the treating physiotherapist to ensure uniform diagnosis; interventions and educational information are correctly delivered to patients. A study manual including procedures and standardized intervention protocols will also be provided. All three treatment groups will receive three patient-physiotherapist contact treatment sessions, to reduce confounding variables [30]. The ESWT only group will receive the educational information but will not receive specific exercise instruction [31]. The exercise groups will receive physiotherapist guidance on exercise instruction along with written instruction. The physiotherapist will ensure each participant is performing the exercises correctly and answer any questions they have regarding them. The ESWT \& exercise group (1) will perform the exercises prior to receiving ESWT during their week 1 and 3 clinic visits, as there is potential for pain post-ESWT [13]. Participants will be informed that increased heel pain could occur following ESWT or exercise, particularly during the first few weeks. The only co-intervention permitted in all three groups will be the written resource. All other forms of active physiotherapy for PHP must be discontinued. 
Those already wearing orthotics can continue doing so, but new or alteration of orthotics will not be permitted during the study. Participants will also be encouraged not to change their regular shoe wear or activity level during the study $[10]$.

Table 3: Intervention protocols

\begin{tabular}{|l|l|}
\hline Group 1: & $\begin{array}{l}3 \text { sessions of R-ESWT one week apart } \\
\text { over } 3 \text { weeks (2000 impulses with an } \\
\text { energy flux density of } 0.16 \mathrm{~mJ} / \mathrm{mm} 2\end{array}$ \\
and a rate of 8 impulses per second) \\
Strength exercise (HSRT) every $2^{\text {nd }}$ \\
day - Heel raise for 8 repetition \\
maximum for 5 sets. Loading will be \\
progressed by using a backpack with \\
books or weights. Clinic visits: Week \\
$1,2 \& 3$
\end{tabular}




\section{Outcome measures}

Trial procedures and process evaluation outcome data will be the primary outcomes measured to determine procedures of a definitive RCT $[18,19]$. Analysing process evaluation data prior to outcome analysis is recommended to avoid biased interpretation of process data [32]. Data will be collected on recruitment, retention, adherence and fidelity. Acceptability of randomisation, outcome measurement and the interventions will be measured by questionnaires and interviews. Implementation fidelity will be assessed by recording attendance, exercise adherence, intervention checklists and participant diaries and interviews [33]. Implementation fidelity can be defined as the degree to which an intervention is implemented as intended [34]. Fidelity enhances scientific confidence that study outcomes are due to interventions and not implementation variables [35]. Fidelity remains poorly reported in physiotherapy research, with a need for robust protocols to ensure clinical translation from findings [36].

Secondary outcome measures to be evaluated (Table 3 ) have been selected based on applicability, guidelines, psychometric properties and previous research for PHP patients undergoing ESWT and physiotherapy interventions. These patientreported outcome measures will be completed at baseline assessment and at 1month and 6-month follow-up electronically, with participants receiving electronic and postal completion reminders. The primary secondary outcome measure will be the Foot Function Index (FFI) at 1-month follow-up. The FFI is the most common foot specific validated outcome measure used in practice for PHP with good psychometric properties [37]. It has been used in the previous studies on exercise interventions for PHP, which are being implemented in this study [10, 11]. It has also been used in most studies which have combined ESWT and exercise for PHP, and in several of the ESWT placebo-controlled studies [15]. A revised FFI (FFI-R) with a 34-item short form and 68-item long form has recently been developed based on criticisms and has shown good psychometric properties [38]. The FFI-R short form has been found to be equally reliable as the long form but significantly decreases participant response burden [38]. The FFI is also 
recommended by several bodies as a validated physiotherapy PHP outcome measure, such as the American Physical Therapy Association [39].

The FFI consists of 23 self-reported items divided into 3 subcategories based on patient values: pain, disability and activity limitation [38]. The patient scores each question on a scale from 0 (no pain or difficulty) to 10 (worst pain imaginable or so difficult it requires help), that best describes their foot over the past week. The pain subcategory consists of 9 items and measures foot pain in different situations, such as walking barefoot versus walking with shoes. The disability subcategory consists of 9 items and measures difficulty performing various functional activities because of foot problems, such as difficulty climbing stairs. The activity limitation subcategory consists of 5 items and measures limitations in activities because of foot problems, such as staying in bed all day. The scores range from 0 to 230, with 0 reflecting no pain, disability, or activity limitations, with both total and subcategory scores being calculated [38]. The minimal important difference is 7 points for the total scale in PHP interventions [40]. The pain specific subscale (PS-FFI) consisting of 9 items scored $0-10$ is frequently chosen on its own in studies investigating physiotherapy for PHP and has high validity and reliability $[38,15]$. The changes in the numeric scores for the first two items on the pain subscale are often used as separate measures, as they are considered to represent the primary concerns articulated by patients with PHP. In clinical practice, patients routinely complain about the severe pain with the first steps in the morning (item 2) and focus on the pain when it is at its worst (item 1). Consequently, some studies have analysed these items separately since these are thought to be most clinically relevant to the patients' PHP complaints [10]. Other foot outcome measures often include subcategories that have little clinical relevance to patients with chronic PHP, unlike the FFI [40].

The Foot and Ankle Ability Measure (FAAM) is a 29-item questionnaire divided into two subscales: 21-item Activities of Daily Living (ADL) Subscale and the, 8-item Sports Subscale. The Sports subscale assesses more difficult tasks that are essential to sport, it is a population-specific subscale designed for 
athletes [41]. Each item is scored on a 5-point Likert scale (4 to 0 ) from 'no difficulty at all' to 'unable to do'. Item score totals, which range from 0 to 84 for the ADL subscale and 0 to 32 for the Sports subscale, are transformed to percentage scores. Higher scores represent higher levels of function for each subscale, with $100 \%$ representing no dysfunction. The minimal clinically important differences are 8 and 9 points for the ADL and Sports subscales, respectively [42]. The FAAM is the only outcome measure which has been validated in a physiotherapy setting specifically with the PHP population [43]. The FAAM has been validated for test content, internal structure, score stability and responsiveness, with Test-retest reliability of 0.89 and 0.87 for the ADL and sports subscales [44]. Evidence for content validity, construct validity, reliability, and responsiveness were obtained among 243 subjects who were separated into a group expected to change and a group expected to remain stable [44]. Both the FFI and FAAM are recommended outcome measures for studies investigating physiotherapy specific interventions for PHP [39]. However, unlike the FAAM, the FFI may be inappropriate for highly active or athletic individuals, functioning above the level of independence in ADLs [45].

Pain visual analogue scale (VAS) scores and the Roles and Maudsley 4-point scale have emerged as the standard outcome measures in ESWT studies to measure pain and function [46]. Although the VAS pain score has good reliability for chronic musculoskeletal disorders, it has questionable validity [47]. A change of $9 \mathrm{~mm}$ on a $100 \mathrm{~mm}$ scale is considered clinically relevant for patients with PHP (Johannsen et al. 2018). The Roles and Maudsley 4-point scale for pain and function is commonly used in ESWT studies [46]. Although its psychometric properties have not been studied it is simple to use and would place the study well in the ESWT PHP literature and allow for outcome comparisons with previous studies. The EuroQol 5-dimension (EQ-5D-5L) is a common global health questionnaire with good validity when tested across several populations to measure QoL. It is also recommended by the NHS to measure health related QoL, so should be included as a QoL measure [48]. Using a Global Rating of Change score will also help determine participants overall satisfaction following completing the interventions. 
Table 4: Secondary outcome measures

\begin{tabular}{|l|}
\hline Secondary outcome measures assessing pain, function and QoL \\
\hline Foot Function Index Revised (FFI-R) \\
\hline Foot and Ankle Ability Measure (FAAM) score \\
\hline RAS pain scores on 100 mm scale \\
\hline Roles \& Maudsley 4-point scale for pain and function \\
\hline EQ-5D-5L QoL score
\end{tabular}

\section{Data processing and analysis}

Outcome measures will be completed on paper or electronically by participants then transferred to excel and SPSS for analysis by the researcher. Quantitative data about the intervention implementation will be analysed using descriptive statistics. Data will be collected on recruitment rates, retention, numbers randomised, adherence, compliance, fidelity, drop-outs and secondary outcome measure completion. Data will be analysed as percentages for categorical data and mean standard deviation for continuous data. Data findings will determine process evaluation and trial procedures of a full-scale RCT. Exploratory inferential analyses of the secondary outcome measures will allow calculation of effect sizes to produce sample size calculations for the full-scale RCT. 


\section{Qualitative interviews}

Following intervention completion, selected patients from each group will undergo semi-structured telephone interviews to cover issues related to trial procedures and acceptability. Although the absence of visual cues via telephone is thought to limit non-verbal data and compromise rapport, there is a lack of evidence showing they produce lower-quality data [49]. Participants may also feel more relaxed and comfortable disclosing information via telephone allowing rich narrative data collection [50]. Qualitative semi-structured interviews allow participants to express their views and experiences in rich detail, while maintaining some structure to address the study objectives [51]. Purposive sampling will be used to select a broad range of patients from each group based to ensure a broad range of perspectives [52]. An estimated 12 participants will be selected based on sampling criteria of age ( 3 age bands) and gender, with two participants in each category. Purposive sampling along dimensions expected to influence complex interventions is recommended for interviews within process evaluations [53].

The interviews will address issues related to process evaluation, intervention fidelity and study procedures. Acceptability to randomisation, outcome measure burden, the interventions and trial participation will be assessed. Collecting qualitative data can also help in developing acceptable treatment fidelity protocols and identifying barriers and implementation strategies [54]. Personal and contextual factors which may affect outcomes will also be explored, such as barriers, intervention expectations and acceptability [55]. Interviews will be recorded using a digital voice recorder, fully anonymised and transcribed verbatim [56]. Data will be analysed using the framework method of analysis and analysis will involve data familiarisation, identifying important themes and issues to develop a thematic framework. Data will be indexed according to the framework leading to a charting of data according to the thematic framework [51]. Mapping and interpreting the themes will help to clarify concepts and help understand and explain the data in relation to the study objectives [57]. Confidentiality risk can be higher in qualitative studies using direct quotes, particularly those involving small specialized sub-populations with a specific pathology in one geographical 
region [58]. To minimize risk, all data collected will be anonymised and participants will be referred to as numbers for quotations.

\section{Discussion}

\section{Key implications/value of the research}

The burden of musculoskeletal disorders on society is increasing along with the need for evidence-based treatments. One of the most common musculoskeletal disorders is PHP, affecting $10 \%$ of the general population, causing significant pain and impaired function. Recently ESWT has emerged as an effective treatment for some musculoskeletal disorders, with evidence for its effectiveness on PHP pain and function, demonstrated by several high-quality RCTs. However, RCTs have compared ESWT with placebo, with no studies comparing its effectiveness with an evidence based HSRT protocol. Recent RCTs have also found that plantar fascia specific HSRT has positive outcomes for PHP. It could therefore be hypothesised that combining these effective treatments could lead to superior outcomes.

In clinical practice, exercise is the most common treatment for PHP and when ESWT is used, it is often combined with exercise. Subsequently, there is a need to ascertain whether the addition of ESWT to physiotherapy exercise leads to better outcomes compared to either of these interventions alone. A three-arm RCT comparing these groups would be the ideal way to investigate this question. A pilot RCT could test recruitment, retention, trial procedures and process evaluation for a definitive RCT. Patients expectations, feasibility and acceptability of ESWT and exercise for PHP also remain unknown. Therefore, the addition of qualitative interviews in a mixed methods pilot RCT would help ascertain acceptability and help explain the intervention outcomes. This pilot study would be the basis for a larger-scale RCT which would be adequately powered to determine the effectiveness of these interventions and determine which is the most effective. 
These findings would then allow for clinical physiotherapy recommendations to be made for the optimal treatment method of this common debilitating condition.

\section{Limitations of the project}

There may be a lack of uptake in patient recruitment for this project, however, recruiting from private clinics, the general population and the NHS if required should ensure that the target of 48 patients is met.

\section{Ethical issues}

There are no major ethical issues anticipated in this project. If recruitment is expanded from private practice to also include NHS patients and staff, then NHS ethics (IRAS) will be sought after gaining local ethical approval. Patients will be identified by physiotherapy staff and sent out a recruitment pack (invitation letter along with participant information sheet). Interested patients will then contact the researchers if they wish to take part in the project. All quantitative and qualitative data collected will be securely stored on drives accessed via password-protected computers in accordance with the Data protection Act (1998). Confidentiality for participants will be ensured throughout the project and all participants will be assigned an ID code. Data will therefore be re-identifiable; however, the code sheet will always be stored separately from participant data.

Funding: No sources of funding were used to assist in the preparation of this article.

Conflicts of interest/Competing interests: The author declares no conflicts of interest relevant to the content of this review.

Authorship contributions: IB conceptualised the work, wrote the first draft of the manuscript, revised the manuscript and approved the final manuscript. 


\section{References}

1. March L, Smith EU, Hoy DG, et al. Burden of disability due to musculoskeletal (MSK) disorders. Best Pract Res Clin Rheumatol 2014;28:353-66.

2. Sebbag E, Felten R, Sagez F, Sibilia J, Devilliers H, Arnaud L. The world-wide burden of musculoskeletal diseases: a systematic analysis of the World Health Organization Burden of Diseases Database. Ann Rheum Dis 2019;78:844-8.

3. Murray CJ, Richards MA, Newton JN, et al. UK health performance: findings of the Global Burden of Disease Study 2010. Lancet 2013;381:997-1020.

4. Trojian T, Tucker AK. Plantar Fasciitis. Am Fam Physician 2019;99:744-50.

5. Monteagudo M, de Albornoz PM, Gutierrez B, Tabuenca J, Alvarez I. Plantar fasciopathy: A current concepts review. EFORT Open Rev 2018;3:485-93.

6. Riel H, Jensen MB, Olesen JL, Vicenzino B, Rathleff MS. Self-dosed and predetermined progressive heavy-slow resistance training have similar effects in people with plantar fasciopathy: a randomised trial. J Physiother 2019;65:14451.

7. Hansen L, Krogh TP, Ellingsen T, Bolvig L, Fredberg U. Long-Term Prognosis of Plantar Fasciitis: A 5- to 15-Year Follow-up Study of 174 Patients With Ultrasound Examination. Orthop J Sports Med 2018;6:2325967118757983.

8. Salvioli S, Guidi M, Marcotulli G. The effectiveness of conservative, nonpharmacological treatment, of plantar heel pain: A systematic review with metaanalysis. Foot (Edinb) 2017;33:57-67.

9. Babatunde OO, Legha A, Littlewood C, et al. Comparative effectiveness of treatment options for plantar heel pain: a systematic review with network metaanalysis. Br J Sports Med 2019;53:182-94. 
10. Digiovanni BF, Nawoczenski DA, Malay DP, et al. Plantar fascia-specific stretching exercise improves outcomes in patients with chronic plantar fasciitis. A prospective clinical trial with two-year follow-up. J Bone Joint Surg Am $2006 ; 88: 1775-81$.

11. Rathleff MS, Molgaard CM, Fredberg $U$, et al. High-load strength training improves outcome in patients with plantar fasciitis: A randomized controlled trial with 12-month follow-up. Scand J Med Sci Sports 2015;25:e292-300.

12. d'Agostino MC, Craig K, Tibalt E, Respizzi S. Shock wave as biological therapeutic tool: From mechanical stimulation to recovery and healing, through mechanotransduction. Int J Surg 2015;24:147-53.

13. Roerdink RL, Dietvorst $M$, van der Zwaard $B$, van der Worp $H$, Zwerver J. Complications of extracorporeal shockwave therapy in plantar fasciitis: Systematic review. Int J Surg 2017;46:133-45.

14. Schmitz C, Csaszar NB, Rompe JD, Chaves H, Furia JP. Treatment of chronic plantar fasciopathy with extracorporeal shock waves (review). J Orthop Surg Res $2013 ; 8: 31,799 X-8-31$.

15. Rompe JD, Furia J, Cacchio A, Schmitz C, Maffulli N. Radial shock wave treatment alone is less efficient than radial shock wave treatment combined with tissue-specific plantar fascia-stretching in patients with chronic plantar heel pain. Int J Surg 2015;24:135-42.

16. Johannsen FE, Herzog RB, Malmgaard-Clausen NM, Hoegberget-Kalisz M, Magnusson SP, Kjaer M. Corticosteroid injection is the best treatment in plantar fasciitis if combined with controlled training. Knee Surg Sports Traumatol Arthrosc 2019;27:5-12.

17. Reilly JM, Bluman E, Tenforde AS. Narrative Review on the Effect of Shockwave Treatment for Management of Upper and Lower Extremity Musculoskeletal Conditions. PM R 2018;

18. Craig $P$, Dieppe $P$, Macintyre $S$, et al. Developing and evaluating complex interventions: the new Medical Research Council guidance. BMJ 2008;337:a1655. 
19. Moore GF, Audrey S, Barker M, et al. Process evaluation of complex interventions: Medical Research Council guidance. BMJ 2015;350:h1258.

20. Hicks C. Research methods for clinical therapists: applied project design and analysis. 5th ed. Edinburgh: Churchill Livingstone/Elsevier; 2009.

21. Arain M, Campbell MJ, Cooper CL, Lancaster GA. What is a pilot or feasibility study? A review of current practice and editorial policy. BMC Med Res Methodol $2010 ; 10: 67,2288-10-67$.

22. Lannin NA, Ada L, Levy $T$, et al. Intensive therapy after botulinum toxin in adults with spasticity after stroke versus botulinum toxin alone or therapy alone: a pilot, feasibility randomized trial. Pilot Feasibility Stud 2018;4:82,018-0276-6. eCollection 2018.

23. Gupta A, Calfas KJ, Marshall SJ, et al. Clinical trial management of participant recruitment, enrollment, engagement, and retention in the SMART study using a Marketing and Information Technology (MARKIT) model. Contemp Clin Trials 2015;42:185-95.

24. Wilsey B, Deutsch R, Marcotte TD. Maintenance of Blinding in Clinical Trials and the Implications for Studying Analgesia Using Cannabinoids. Cannabis Cannabinoid Res 2016;1:139-48.

25. Suresh K, Thomas SV, Suresh G. Design, data analysis and sampling techniques for clinical research. Ann Indian Acad Neurol 2011;14:287-90.

26. Efird J. Blocked randomization with randomly selected block sizes. Int J Environ Res Public Health 2011;8:15-20.

27. Lou J, Wang S, Liu S, Xing G. Effectiveness of Extracorporeal Shock Wave Therapy Without Local Anesthesia in Patients With Recalcitrant Plantar Fasciitis: A Meta-Analysis of Randomized Controlled Trials. Am J Phys Med Rehabil 2017;96:529-34.

28. McLean SM, Burton M, Bradley L, Littlewood C. Interventions for enhancing adherence with physiotherapy: a systematic review. Man Ther 2010;15:514-21. 
29. Gerdesmeyer L, Frey C, Vester J, et al. Radial extracorporeal shock wave therapy is safe and effective in the treatment of chronic recalcitrant plantar fasciitis: results of a confirmatory randomized placebo-controlled multicenter study. Am J Sports Med 2008;36:2100-9.

30. Suttanon $P$, Hill KD, Said CM, et al. Feasibility, safety and preliminary evidence of the effectiveness of a home-based exercise programme for older people with Alzheimer's disease: a pilot randomized controlled trial. Clin Rehabil 2013;27:42738.

31. Patten CA, Bronars CA, Vickers Douglas KS, et al. Supervised, Vigorous Intensity Exercise Intervention for Depressed Female Smokers: A Pilot Study. Nicotine Tob Res 2017;19:77-86.

32. Oakley A, Strange V, Bonell C, Allen E, Stephenson J, RIPPLE Study Team. Process evaluation in randomised controlled trials of complex interventions. BMJ $2006 ; 332: 413-6$.

33. Swindle T, Selig JP, Rutledge JM, Whiteside-Mansell L, Curran G. Fidelity monitoring in complex interventions: a case study of the WISE intervention. Arch Public Health 2018; 76:53,018-0292-2. eCollection 2018.

34. Gearing RE, El-Bassel N, Ghesquiere A, Baldwin S, Gillies J, Ngeow E. Major ingredients of fidelity: a review and scientific guide to improving quality of intervention research implementation. Clin Psychol Rev 2011;31:79-88.

35. Borrelli B. The Assessment, Monitoring, and Enhancement of Treatment Fidelity In Public Health Clinical Trials. J Public Health Dent 2011;71:S52-63.

36. Toomey E, Hardeman W. Addressing Intervention Fidelity Within Physical Therapy Research and Clinical Practice. J Orthop Sports Phys Ther 2017;47:8958.

37. SooHoo NF, Samimi DB, Vyas RM, Botzler T. Evaluation of the validity of the Foot Function Index in measuring outcomes in patients with foot and ankle disorders. Foot Ankle Int 2006;27:38-42. 
38. Budiman-Mak E, Conrad KJ, Mazza J, Stuck RM. A review of the foot function index and the foot function index - revised. J Foot Ankle Res 2013;6:5,1146-6-5.

39. Martin RL, Davenport TE, Reischl SF, et al. Heel pain-plantar fasciitis: revision 2014. J Orthop Sports Phys Ther 2014;44:A1-33.

40. Landorf KB, Radford JA. Minimal important difference: Values for the Foot Health Status Questionnaire, Foot Function Index and Visual Analogue Scale. The Foot 2008;18:15-9.

41. Walmsley $S$, Williams $A E$, Ravey $M$, Graham $A$. The rheumatoid foot: $a$ systematic literature review of patient-reported outcome measures. J Foot Ankle Res $2010 ; 3: 12,1146-3-12$.

42. Martin RL, Irrgang JJ. A survey of self-reported outcome instruments for the foot and ankle. J Orthop Sports Phys Ther 2007;37:72-84.

43. McPoil TG, Martin RL, Cornwall MW, Wukich DK, Irrgang JJ, Godges JJ. Heel pain--plantar fasciitis: clinical practice guildelines linked to the international classification of function, disability, and health from the orthopaedic section of the American Physical Therapy Association. J Orthop Sports Phys Ther 2008;38:A1A18.

44. Martin RL, Irrgang JJ, Burdett RG, Conti SF, Van Swearingen JM. Evidence of validity for the Foot and Ankle Ability Measure (FAAM). Foot Ankle Int $2005 ; 26: 968-83$.

45. Agel J, Beskin JL, Brage $M$, et al. Reliability of the Foot Function Index: $A$ report of the AOFAS Outcomes Committee. Foot Ankle Int 2005;26:962-7.

46. Gollwitzer $H$, Saxena A, DiDomenico LA, et al. Clinically relevant effectiveness of focused extracorporeal shock wave therapy in the treatment of chronic plantar fasciitis: a randomized, controlled multicenter study. J Bone Joint Surg Am 2015;97:701-8. 
47. Boonstra AM, Schiphorst Preuper HR, Reneman MF, Posthumus JB, Stewart RE. Reliability and validity of the visual analogue scale for disability in patients with chronic musculoskeletal pain. Int J Rehabil Res 2008;31:165-9.

48. Herdman M, Gudex C, Lloyd A, et al. Development and preliminary testing of the new five-level version of EQ-5D (EQ-5D-5L). Qual Life Res 2011;20:1727-36.

49. Novick G. Is there a bias against telephone interviews in qualitative research? Res Nurs Health 2008;31:391-8.

50. Mealer M, Jones $\mathrm{Rn}$ J. Methodological and ethical issues related to qualitative telephone interviews on sensitive topics. Nurse Res 2014;21:32-7.

51. Ritchie J, Lewis J, McNaughton Nicholls C, Ormston R. Qualitative research practice: a guide for social science students and researchers. Second ed. Los Angeles: Sage; 2014.

52. Green J, Thorogood N. Qualitative methods for health research. 4th ed. Los Angeles: Sage; 2018.

53. Munro A, Bloor M. Process evaluation: the new miracle ingredient in public health research? Qualitative Research 2010;10:699-713.

54. Van den Branden S, Van den Broucke S, Leroy R, Declerck D, Hoppenbrouwers $\mathrm{K}$. Evaluating the implementation fidelity of a multicomponent intervention for oral health promotion in preschool children. Prev Sci 2015;16:1-10.

55. Cormier S, Lavigne GL, Choiniere M, Rainville P. Expectations predict chronic pain treatment outcomes. Pain 2016;157:329-38.

56. Brinkmann S, Kvale S. Doing interviews. Los Angeles: Sage; 2018; 2018.

57. Pope C, Ziebland S, Mays N. Qualitative research in health care. Analysing qualitative data. BMJ 2000;320:114-6.

58. Polgar S, Thomas SA. Introduction to research in the health sciences. Sixth ed. Edinburgh: Churchill Livingstone; 2013. 
59. Mann JB, Thyfault JP, Ivey PA, Sayers SP. The effect of autoregulatory progressive resistance exercise vs. linear periodization on strength improvement in college athletes. J Strength Cond Res 2010 Jul;24(7):1718-1723.

60. Zourdos MC, Jo E, Khamoui AV, et al. Modified Daily Undulating Periodization Model Produces Greater Performance Than a Traditional Configuration in Powerlifters. J Strength Cond Res 2016;30:784-91.

61. Zourdos MC, Klemp A, Dolan C, et al. Novel Resistance Training-Specific Rating of Perceived Exertion Scale Measuring Repetitions in Reserve. J Strength Cond Res $2016 ; 30: 267-75$.

62. Fairman CM, Zourdos MC, Helms ER, Focht BC. A Scientific Rationale to Improve Resistance Training Prescription in Exercise Oncology. Sports Med 2017;47:1457-65.

63. Graham T, Cleather DJ. Autoregulation by "Repetitions in Reserve" Leads to Greater Improvements in Strength Over a 12-Week Training Program Than Fixed Loading. J Strength Cond Res 2019; .

64. Helms ER, Cross MR, Brown SR, Storey A, Cronin J, Zourdos MC. Rating of Perceived Exertion as a Method of Volume Autoregulation Within a Periodized Program. J Strength Cond Res 2018;32:1627-36. 